\title{
Lupin seed $\gamma$-conglutin lowers blood glucose in hyperglycaemic rats and increases glucose consumption of HepG2 cells
}

\author{
Maria Rosa Lovati $^{1 *}$, Cristina Manzoni ${ }^{1}$, Silvia Castiglioni ${ }^{1}$, Anna Parolari $^{1}$, Chiara Magni $^{2}$ \\ and Marcello Duranti ${ }^{2}$ \\ ${ }^{1}$ Department of Pharmacological Sciences, Università degli Studi di Milano, 20133 Milano, Italy \\ ${ }^{2}$ Department of Agri-Food Molecular Sciences, Università degli Studi di Milano, 20133 Milano, Italy
}

(Received 12 November 2010 - Revised 10 February 2011 - Accepted 25 March 2011 - First published online 28 June 2011)

\section{Abstract}

The aim of the present study was to evaluate the effect of a chronic oral $\gamma$-conglutin treatment in male Sprague-Dawley rats in which hyperglycaemia had been induced by supplying $10 \%$ D-glucose in drinking-water. A $\gamma$-conglutin dosage of $28 \mathrm{mg} / \mathrm{kg}$ body weight was daily administered to animals for $21 \mathrm{~d}$. Plasma glucose, insulin and glucose overloading were monitored. Chronic administration of glucose resulted in a statistically significant $(P<0 \cdot 05)$ increase in fasting blood glucose $(2 \cdot 5$-fold $)$ and insulin $(2 \cdot 7$-fold $) v$. the values recorded in control rats. Simultaneous treatment with $\gamma$-conglutin attenuated the rise in plasma glucose (1.9-fold) and insulin (1.8-fold) levels in the glucose-fed rats $(P<0 \cdot 05)$. Fasting insulin and homeostasis model of insulin resistance were decreased by 34 and $48 \%(P<0 \cdot 05)$, respectively, in the $\gamma$-conglutin-treated rats $v$. the values found in pair-fed animals. To confirm these results with a different approach, HepG2 cells, grown for 24 and $48 \mathrm{~h}$ in Dulbecco's minimum essential medium containing different glucose concentrations (5.5, $11 \cdot 1$ and $16.5 \mathrm{mmol} / \mathrm{l}$ ), were exposed to $10 \mu \mathrm{mol} / \mathrm{l} \gamma$-conglutin with or without $10 \mathrm{mmol} / \mathrm{l}$ metformin or $100 \mathrm{nmol} / \mathrm{l}$ insulin. $\gamma$-Conglutin increased glucose consumption (from 1.5- to 2.5-fold) in HepG2 cells, under all experimental conditions; this effect was more evident after $48 \mathrm{~h}$ incubation. Moreover, in this in vitro model, the addition of $\gamma$-conglutin potentiated the activity of insulin and metformin in cell glucose consumption. These findings extend the previous ones and suggest the potential use of lupin $\gamma$-conglutin in the control of glycaemia.

Key words: Hyperglycaemic rats: Lupinus albus seed protein: $\gamma$-Conglutin: HepG2 cells: Glucose consumption

A number of studies on the beneficial effects of plant-derived food proteins have appeared in recent years. Most of these studies evidence the biological activities of some legume seed storage proteins or fragments thereof and those of other cotyledonary proteins on the markers of chronic diseases, such as lipid disorders, diabetes, hypertension and cancer, which are typical of industrialised societies ${ }^{(1-4)}$. Although the beneficial effects of legume seed dietary intake are the basis for various health claims, some questions still remain to be solved, including the identification of the biologically active peptides, their metabolic fate and mechanism of action, in order to identify the potential of these molecules both in the nutraceutical industry and in drug companies.

Many papers from our groups pointed out the positive effects of soya and white lupin proteins on lipid and glucose metabolism. Investigations on soya polypeptides, namely the purified $\alpha^{\prime}$ subunit from $7 \mathrm{~S}$ globulin ${ }^{(5)}$, a recombinant polypeptide including $\alpha^{\prime}$ subunit extension region ${ }^{(6)}$ and peptides from the $\beta$ subunit $^{(7)}$, have been reported. On the other hand, new data on the potential of $\gamma$-conglutin from lupin seed on glucose metabolism control are emerging ${ }^{(8)}$.

$\gamma$-Conglutin is a cotyledonary white lupin (Lupinus albus, L.) seed glycoprotein with molecular weight of approximately $47 \mathrm{kDa}$, composed of two disulphide bound heterogeneous subunits of 29 and $17 \mathrm{kDa}^{(9)}$. Its amount in the lupin seeds ranges from 3 to $5 \%{ }^{(10)}$. Proteins having sequence homology with $\gamma$-conglutin, for which the biological function has not been assessed yet, have also been found in other plant species, including soya ${ }^{(11)}$ and carrot ${ }^{(12)}$.

In a previous paper, the in vitro interaction of $\gamma$-conglutin with mammalian insulin ${ }^{(13)}$, as the homologous soya basic $7 \mathrm{~S}$ protein also does ${ }^{(14)}$, was described, and the kinetic and thermodynamic parameters of binding were also measured. In parallel, the glucose-lowering effect of this protein in normal rats, upon glucose overload trial, was described for the first time. In that study ${ }^{(13)}$, the protein was administered by gavage half an hour before glucose administration, and glycaemia was measured at $30 \mathrm{~min}$ intervals. In $\boldsymbol{\gamma}$-conglutin-treated rats, a dose-dependent statistically significant reduction in glycaemia

Abbreviations: BW, body weight; DMEM, Dulbecco's minimum essential medium; HOMA, homeostasis model assessment; IR, insulin resistance.

*Corresponding author: M. R. Lovati, fax +39250318284, email mariarosa.lovati@unimi.it 
was detected, comparable with that of about half a dose of metformin.

Nevertheless, these previous findings need to be confirmed in in vivo models of pre-diabetic or diabetic state in order to assess $\gamma$-conglutin actual potential. On this basis, the aim of the present study was to evaluate the effect of a chronic oral administration of $\gamma$-conglutin in male Sprague-Dawley rats in which hyperglycaemia was induced by supplying $10 \%$ D-glucose in drinking-water ${ }^{(15)}$ in addition to a normal chow diet during 3 weeks. In this animal model, chronic glucose feeding resulted in non-insulin-dependent diabetes as reflected by the increase in both blood glucose and insulin levels. In addition to these trials, further experiments were carried out on HepG2 cells, a human hepatoma cell line, to assess $\gamma$-conglutin effects on glucose consumption and to get hints on the possible mechanism.

\section{Materials and methods}

\section{Materials}

Dry mature seeds of white lupin (L. albus L, var. Multitalia) were kindly provided by Dr Massimo Fagnano, University of Naples, Naples, Italy. Type F, a $\boldsymbol{\gamma}$-conglutin-enriched lupin protein isolate, prepared as described in Bez et al. ${ }^{(16)}$, was kindly supplied by Fraunhofer Institute (Munich, Germany). This preparation, the composition of which is detailed in the cited reference, contained about $80 \%$ protein and was used as such in the in vivo experiments.

\section{White lupin $\gamma$-conglutin purification}

A laboratory-scale purified $\gamma$-conglutin was utilised in the in vitro assay. The procedure for $\gamma$-conglutin purification, as described by Duranti et al. ${ }^{(17)}$, was slightly modified to improve the homogeneity of the preparation. In particular, after conventional chromatographic steps, which included gel permeation chromatography, ion exchange chromatography on both Whatman DE52 diethylaminoethyl-cellulose and carboxymethylcellulose, a further step of metal affinity chromatography was added. For this purpose, the protein solution was loaded onto a nickel column (NiNTA-Agarose; Qiagen, Milan, Italy) equilibrated in $50 \mathrm{~mm}$-Tris- $\mathrm{HCl}$, pH $7 \cdot 4$, containing $0.5 \mathrm{M}-\mathrm{NaCl}$. The protein bound to the matrix was subsequently eluted with $50 \mathrm{~mm}$ sodium acetate, $\mathrm{pH} 4.5$, containing $0.5 \mathrm{M}-\mathrm{NaCl}$. The purified protein was desalted by dialysis against MilliQ water and freeze dried.

For the estimation of purified $\gamma$-conglutin concentrations, optical measurements at $280 \mathrm{~nm}$ were made. The extinction coefficient of 0.733 for a solution of $1 \mathrm{mg} / \mathrm{ml}$ was used ${ }^{(18)}$.

SDS-PAGE was carried out on $12 \%$ polyacrylamide gels, according to Laemmli ${ }^{(19)}$ under reducing conditions using a mini-Protean II cell (Bio-Rad, Milan, Italy). The sample buffer contained $0.25 \mathrm{~m}$-Tris-HCl, $\mathrm{pH} 6 \cdot 8,7 \cdot 5 \%$ glycerol, $2 \%$ SDS and $5 \%$ mercaptoethanol. Samples were heated at $100^{\circ} \mathrm{C}$ for 5 min before loading. The gels were stained with Coomassie Blue. Densitometric scanning of the gel was carried out by ImageMaster 1D software (Amersham Pharmacia Biotech, Milan, Italy).

\section{Animals, treatments and homeostasis model assessment index}

Male Sprague-Dawley CD rats (Charles River Laboratories Italia Srl, Calco-Lecco, Italy), body weight (BW) 150-175 g, were housed in a room with controlled lighting $(12 \mathrm{~h} / \mathrm{d})$, constant temperature $\left(18^{\circ} \mathrm{C}\right)$ and relative humidity $(55-$ $65 \%$ ). Following a $2 \mathrm{~d}$ adaptation period, during which they were fed a standard diet (pelleted commercial non-purified diet - Mucedola 4RF21; Settimo Milanese, Milan, Italy), the animals were given $10 \% \mathrm{D}$-glucose in drinking-water for the entire experimental period. The rats had free access to water. The mean daily water intake was between 20 and $30 \mathrm{ml} / \mathrm{rat}$, estimating an average daily intake of D-glucose about $2-3 \mathrm{~g}$. After 1 week, the animals were divided in two groups of twelve rats according to their BW and plasma glucose concentrations, so that the distribution between the groups was similar. The animals were daily treated (at 09.00 hours) by gavage for 3 weeks as follows: one group (D-glucose-treated) received only the vehicle (1\% carboxymethylcellulose), whereas the other group (D-glucose $+\gamma$ conglutin) received $100 \mathrm{mg} / \mathrm{kg} \mathrm{BW}$ type $\mathrm{F}$, corresponding to $28 \mathrm{mg} / \mathrm{kg} \mathrm{BW} \boldsymbol{\gamma}$-conglutin in carboxymethylcellulose. A third group of rats received only the vehicle without D-glucose in drinking-water (controls). Food intake and BW were monitored weekly. At the end of treatment, glucose was determined by an enzymatic method (Sigma-Aldrich, Milan, Italy), and fasting insulin was evaluated using a LINCO Rat Insulin RIA kit (LINCO, Millipore, Bedford, MA, USA) on plasma of overnight fasted animals.

At the end of the experimental period, glucose loading was carried out on $10 \mathrm{~h}$ fasted animals. At time 0 , each rat was given $2 \mathrm{~g} / \mathrm{kg}$ BW D-glucose, administered orally. Blood samples were withdrawn from the tail vein of each rat under light diethyl ether anesthesia at 30, 60, 120 and $180 \mathrm{~min}$ following the carbohydrate load. Aliquots of serum were stored at $-20^{\circ} \mathrm{C}$ until assayed. Glucose was determined as described earlier.

To estimate the degree of insulin resistance (IR), the homeostasis model assessment (HOMA) was used as an index of IR according to Midaoui \& De Champlain ${ }^{(20)}$. The HOMA-IR index was calculated using the following equation:

$$
\text { fasting insulin }(\mu \mathrm{U} / \mathrm{ml}) \times \text { fasting glucose }(\mathrm{mmol} / \mathrm{l}) / 22.5 \text {. }
$$

All procedures involving animals and their care were conducted in accordance with institutional guidelines that are in compliance with national (D.L. no. 116, G.U. Suppl. 40, 18 February 1992, Circolare no. 8, G.U. July 1994) and international laws and policies (EEC Council Directive 86/609, OJL 358, 1, 12 December 1987; Guide for the Care and Use of Laboratory Animals published by the US National Institute of Health-NIH Publication no. 85-23, revised 1996).

\section{Cell cultures and treatments}

All culture reagents, glucose-Trinder kit, 1,1-dimethylbiguanide hydrochloride (metformin) and biosynthetic human insulin were obtained from Sigma-Aldrich. The established human 
hepatoma cell line (HepG2) was obtained from American Type Culture Collection (Rockville, MD, USA). Cells were grown in monolayer in $75 \mathrm{~cm}^{2}$ flasks and maintained at $37^{\circ} \mathrm{C}$ in a humidified atmosphere of $95 \%$ air, $5 \% \mathrm{CO}_{2}$ in Dulbecco's minimum essential medium (DMEM; $5.5 \mathrm{~mm}$ glucose) containing $10 \%$ fetal bovine serum, non-essential amino acids ( $1 \%, \mathrm{v} / \mathrm{v}), 200 \mathrm{~nm}-\mathrm{L}-$ glutamine, penicillin $\left(10^{5} \mathrm{U} / \mathrm{l}\right.$; $0.06 \mathrm{~g} / \mathrm{l})$ and streptomycin $(0.1 \mathrm{~g} / \mathrm{l})$, and of sodium pyruvate $(0 \cdot 11 \mathrm{~g} / \mathrm{l})$. To evaluate glucose consumption, $2 \mathrm{~d}$ before the experiments, cells were seeded in twenty-four-well plates (to give $1.5 \times 10^{5}$ cells/well) with some left blank. After the cells reached confluence, the medium was replaced by DMEM supplemented with $0.2 \%$ bovine serum albumin and glucose at various concentrations $(5 \cdot 5,11 \cdot 1$ and $16 \cdot 5 \mathrm{mmol} / \mathrm{l})$. After $12 \mathrm{~h}$, the medium was removed and the cells grown in bovine serum albumin-DMEM were exposed, under sterile conditions, to $10 \mu \mathrm{mol} / 1 \boldsymbol{\gamma}$-conglutin with or without metformin $(10 \mathrm{mmol} / \mathrm{l})$ and/or insulin $(100 \mathrm{nmol} / \mathrm{l})$, including the blank wells, for 24 or $48 \mathrm{~h}$.

At the end of incubation periods, the medium was removed, and glucose concentrations were determined as described previously (Sigma-Aldrich). The glucose concentration of the wells with cells was subtracted from the glucose of the blank wells to obtain the amount of glucose consumption ${ }^{(21)}$. To assess cell viability, culture media from cells exposed to the different compounds were tested by methyltetrazolium salts assay, essentially as described by Lovati et $a l^{(1)}$.

\section{Statistical analyses}

Results are reported as means with their standard errors of the mean. Statistical analyses of the individual differences in plasma glucose, insulin, HOMA-IR and in cell glucose consumption were carried out by one-way ANOVA with Bonferroni's post hoc test. Results of all analyses were considered to be statistically significantly different at $P<0.05$.

\section{Results}

\section{Analysis of laboratory-purified and pilot plant-enriched} $\gamma$-conglutin preparations for the in vitro and in vivo assays

Fig. 1 shows the SDS-PAGE profile of the two $\gamma$-conglutin preparations used in the present study for trials with rats and in cell studies, respectively. Under non-reducing conditions, one major band of about $50 \mathrm{kDa}$ was visible in the laboratory-scale purified protein. When reducing conditions were applied, the profile corresponded to the typical pattern of $\gamma$-conglutin ${ }^{(22)}$ with one subunit of about $30 \mathrm{kDa}$ and another of $17 \mathrm{kDa}$. This sample appeared to be homogenous enough to ensure the reliability of the results in the assays with cell models. On the other hand, pilot plant $\gamma$-conglutin-enriched preparation showed a number of contaminant bands in SDS-PAGE (Fig. 1(a) and (b), lane 1). Nevertheless, the bands corresponding to $\gamma$-conglutin were present as well. Therefore, in order to determine the amount of $\gamma$-conglutin in this product for the in vivo dosages, densitometric scanning of the corresponding lanes was carried out

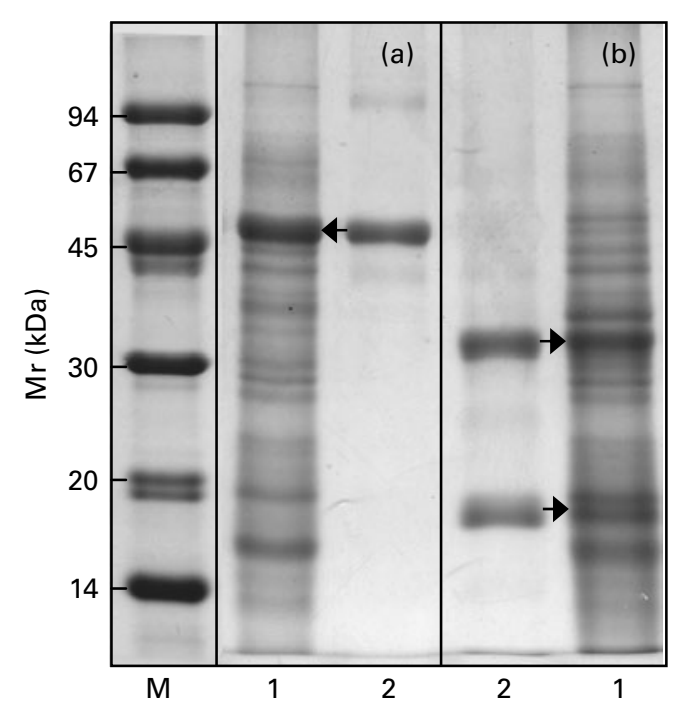

Fig. 1. SDS-PAGE under (a) non-reducing and (b) reducing conditions of $\gamma$-conglutin preparations used in the present study. The gels were stained by Coomassie brilliant blue. Sample lanes are as follows: M, marker; 1, type F; 2 , purified $\gamma$-conglutin.

(not shown). According to this analysis, the amount of $\gamma$-conglutin was estimated to be about $35 \%$ of total proteins. Since proteins in the preparation were about $80 \%$ of the dry weight, these data allowed calculation of a percentage of $28 \mathrm{mg} / \mathrm{kg} \mathrm{BW} \gamma$-conglutin in the preparation that was daily administered to rats over 3 weeks.

\section{$\gamma$-Conglutin administration reduces plasma glucose in the in vivo model of type 2 diabetes}

The dosage of $28 \mathrm{mg} / \mathrm{kg}$ BW $\boldsymbol{\gamma}$-conglutin was selected according to lowest dosage of the previous trials consisting of acute glucose overloads in normal rats ${ }^{(13)}$. BW were not modified either by glucose feeding or by $\gamma$-conglutin treatment in all groups (controls, 244.6 (SE 3.8) g; glucose-treated, 243.7 (SE 5.5) g; glucose-treated $+\gamma$-conglutin, 241.00 (SE 8.0) g). As depicted in Fig. 2(a), chronic glucose administration resulted in a statistically significant $(P<0 \cdot 01)$ increase in fasting blood glucose (2.5-fold); conversely, simultaneous treatment with $\gamma$-conglutin attenuated the rise in glucose ( 1.9 -fold) so that the glucose levels in these animals were reduced by $22 \%$ in comparison with those recorded in the glucose-treated rats. In glucose-drinking rats, insulin levels increased by $170 \%$ (2.7-fold) (Fig. 2(b); $P<0.05$ ); the treatment with $\gamma$-conglutin reduced this increase to $79 \%(P<0.05)$, although the levels remained higher than in control animals. Chronic glucose feeding increased the IR index, as expressed by HOMA-IR, by $582 \%$ (Fig. $2(\mathrm{c}) ; P<0 \cdot 05$ ). $\gamma$-Conglutin treatment attenuated this increase by $252 \%$ in glucose-fed rats, and the comparison between the HOMA-IR indexes in the two groups of rats (glucose-treated and untreated) showed an improvement in IR by $48 \%(P<0.05)$, following daily administration of $\gamma$-conglutin.

The oral glucose tolerance test, carried out at the end of experimental period on all animals enrolled in the study, showed (Fig. 3(a)) a statistically significant reduction 

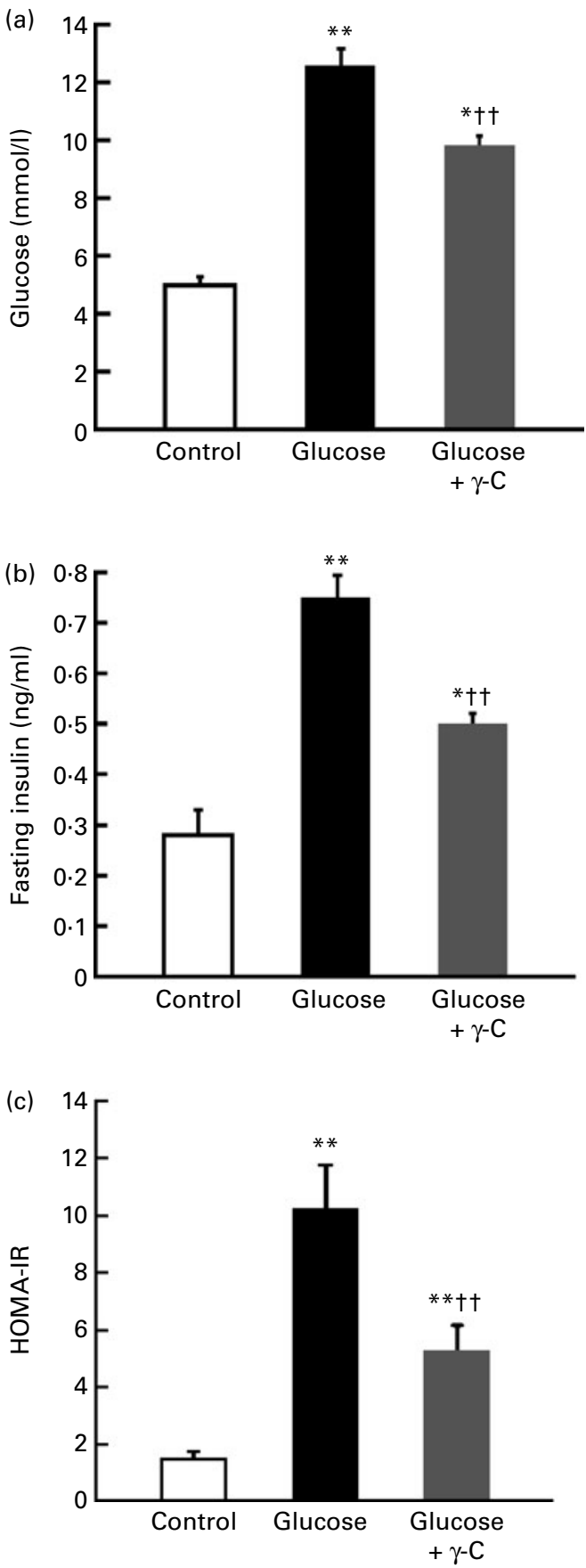

Fig. 2. Effects of chronic glucose drinking, combined with or without $\gamma$-conglutin $(\gamma-\mathrm{C})$ supplementation (a) on plasma glucose levels expressed in $\mathrm{mmol} / \mathrm{l}$, (b) on plasma insulin levels expressed in $\mathrm{ng} / \mathrm{ml}$ and (c) on index of insulin resistance (IR; plasma glucose $\times$ insulin/22.5 = homeostasis model assessment (HOMA)). Values are means, with their standard errors represented by vertical bars, $n 12$. Mean values were significantly different from those of controls: ${ }^{*} P<0.05$, ${ }^{\star *} P<0.01$. Mean values were significantly different from those of glucose group: ${ }^{\dagger \dagger} P<0.01$.

$(-25 \% ; \quad P<0.05)$ of plasma glucose in glucose-treated $\gamma$-conglutin animals in comparison with that obtained in control pair-fed rats. Lower glucose levels were detected in $\gamma$-conglutin-treated animals at $30 \mathrm{~min}$, from glucose overloading, and persisted until $180 \mathrm{~min}$, although glycaemia in these rats was higher than that showed by controls. Moreover, upon 3-week treatment with $\gamma$-conglutin, both fasting blood glucose and postprandial blood glucose $(2 \mathrm{~h})$ were reduced ( -21 and $-12 \%$, respectively; $P<0 \cdot 05)$, suggesting improved insulin sensitivity in the treated animals (Fig. 3(b)).

\section{$\gamma$-Conglutin improved glucose metabolism in vitro}

Glucose consumption was examined in HepG2 cells following incubation with purified $\gamma$-conglutin. The dose of $10 \mu \mathrm{mol} / 1$ $\gamma$-conglutin was selected from previous experiments, where an up-regulation of LDL receptors was detected in HepG2 cells after pre-incubation with $\gamma$-conglutin ${ }^{(4)}$. Cells were grown in DMEM containing different glucose concentrations in order to simulate normal $(5.5 \mathrm{mmol} / \mathrm{l}=990 \mathrm{mg} / \mathrm{l})$ or moderate high glucose $(11 \cdot 1 \mathrm{mmol} / \mathrm{l}=2000 \mathrm{mg} / \mathrm{l})$ and severe hyperglycaemia $(16.5 \mathrm{mmol} / \mathrm{l}=3000 \mathrm{mg} / \mathrm{l})$ in human subjects. Moreover,
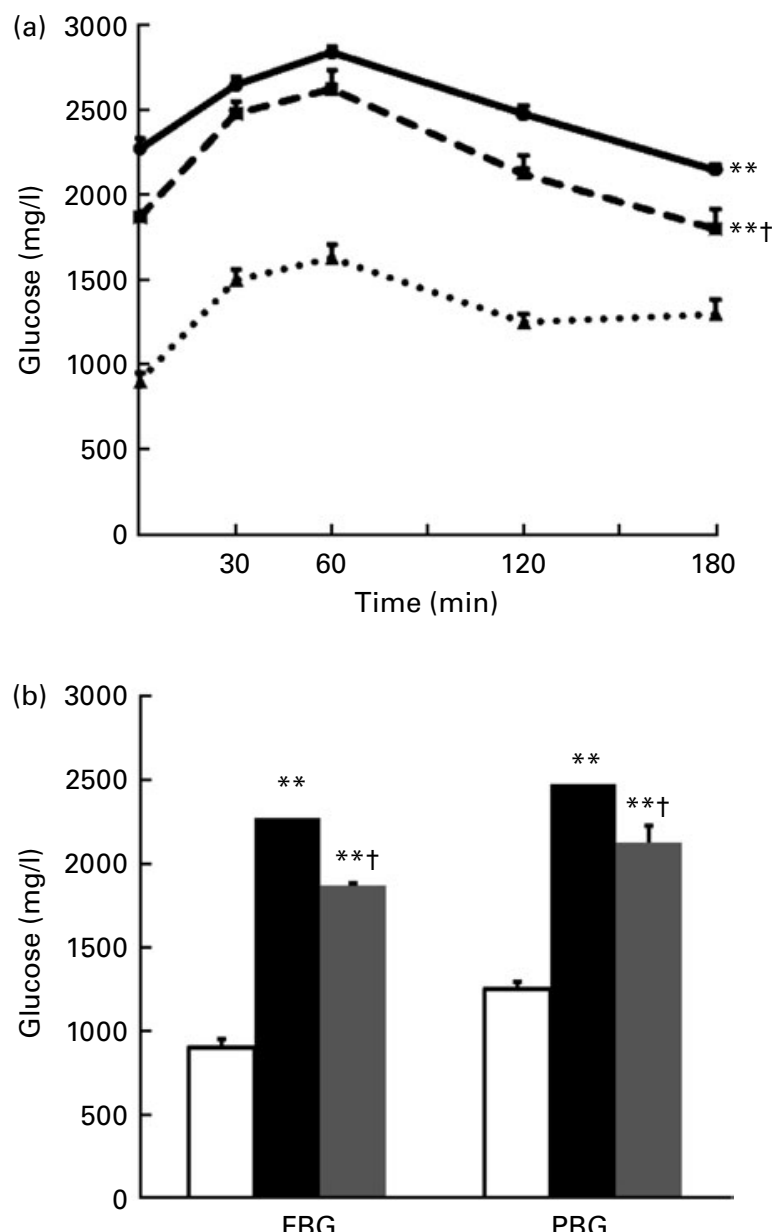

Fig. 3. (a) Influence of $\gamma$-conglutin $(\gamma-C)$ supplementation on plasma glucose concentrations of rats during oral glucose overloading trials $(2 \mathrm{~g} / \mathrm{kg}$ body weight). Plasma glucose was assayed in rats at various times from glucose overload as reported in the 'Materials and methods' section. (b) Fasting blood glucose (FBG) and $2 \mathrm{~h}$ postprandial blood glucose (PBG) determined during glucose overload experiment in the rats. Values are means, with their standard errors represented by vertical bars, $n 12 .{ }^{* \star}$ Mean values were significantly different from those of controls $(P<0.01)$. †Mean values were significantly different from those of glucose group $(P<0.05)$. (a) $\ldots \mathbf{1} \cdots$, Control; $-\longrightarrow$, glucose; $\rightarrow-$, glucose $+\gamma$-C. (b) $\square$, Control; $\square$, glucose; $\square$, glucose $+\gamma-C$. 
insulin $(100 \mathrm{nmol} / \mathrm{l})$ and metformin $(10 \mathrm{mmol} / \mathrm{l})$ were used alone or in combination with $\gamma$-conglutin to assess the potential synergism/antagonism in glucose consumption following 24 or $48 \mathrm{~h}$ incubation.

As depicted in Fig. 4, the addition of insulin or metformin to HepG2 cells grown in DMEM containing different amounts of glucose induced a statistically significant increase $(P<0.05)$ in the glucose consumption after 24 and $48 \mathrm{~h}$ incubation. When the glucose in the culture medium was normal $(5.5 \mathrm{mmol} / \mathrm{l})$, no statistically significant effect on glucose consumption was detected on the addition of $\gamma$-conglutin in all experimental conditions (control, insulin and metformin). On the other hand, the effect of $\gamma$-conglutin in glucose consumption $(P<0.05)$ was evident in cells grown in DMEM with moderate $(11.1 \mathrm{mmol} / \mathrm{l})$ or elevated $(16.5 \mathrm{mmol} / \mathrm{l})$ glucose content; moreover, the concomitant addition of $\gamma$-conglutin to HepG2 cells exposed to insulin or metformin increased further the glucose consumption, normally stimulated by both compounds. In particular, when the glucose concentration in the culture medium increased from 5.5 to $11.1 \mathrm{mmol} / \mathrm{l}$, the glucose expenditure induced by $\gamma$-conglutin, after $24 \mathrm{~h}$ of incubation, was elevated to 100, 60 and 18\%, respectively in controls, insulin- and metformin-treated cells (Fig. 4(b)). When the glucose concentration increased from 11.1 to $16.5 \mathrm{mmol} / \mathrm{l}$, the amount of glucose consumed in $24 \mathrm{~h}$ was enhanced by 109 , 33 and $43 \%$, respectively in controls, insulin- and metformin-treated cells by the addition of $\gamma$-conglutin addition (Fig. 4(c)). This trend was shared by HepG2 cells incubated for $48 \mathrm{~h}$ in the same experimental conditions: the addition of $\gamma$-conglutin induced a statistically significant increase in the amount of glucose consumed as depicted in the Fig. 4((e) and (f)).

The glucose-lowering effect of $\gamma$-conglutin observed in HepG2 cells was not linked to an increment in cell number due to the glucose level, since we did not observe any change in methyltetrazolium salts optical density (data not shown). In addition, the results obtained following the exposure of HepG2 cells to $\boldsymbol{\gamma}$-conglutin were compared for each time and glucose concentration with the respective controls. Moreover, a previous experiment (data not shown), in which mannitol was added to $5.5 \mathrm{mmol} / 1$ glucose DMEM, pointed out that the present results were due to the activity of tested compounds ( $\gamma$-conglutin, insulin and metformin) and not to the effect of hyperosmolarity.

\section{Discussion}

Type 2 diabetes is a chronic metabolic disorder, often characterised by IR, which leads to several secondary complications, including hypertension, atherosclerosis, coronary artery disease and hyperlipidaemia ${ }^{(23)}$. Approximately 150 million people worldwide are affected by the disease at present, with a projection of 300 million people being affected by 2025. Diabetes has become a serious public health problem, particularly in developed countries ${ }^{(24)}$. Research in an effective anti-diabetic agent, in addition to those already available, would be of great interest for the treatment of type 2 diabetes. Legume seeds, due to the nutraceutical potentialities of some
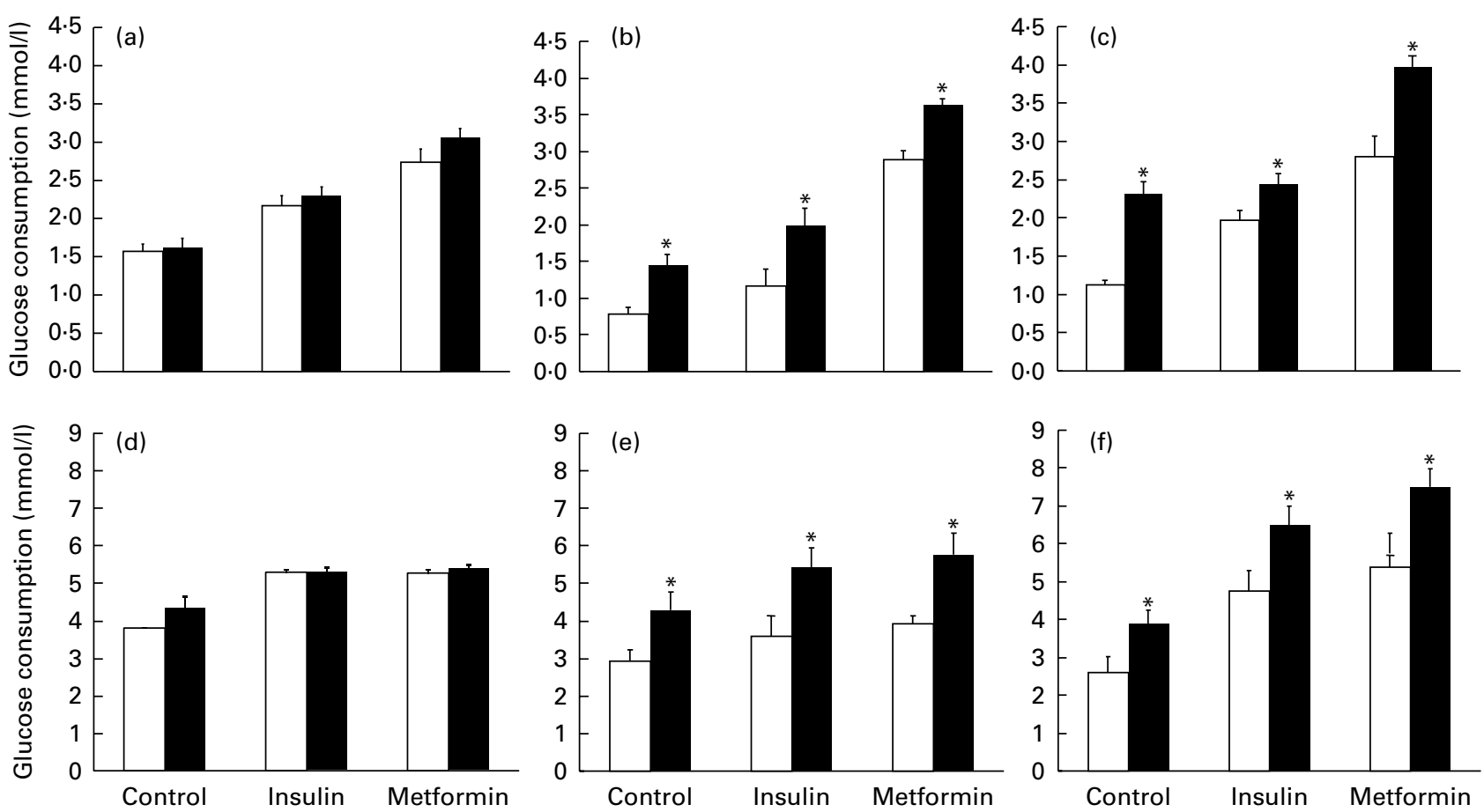

Fig. 4. (a, b, c) Glucose consumption by HepG2 cells after $24 \mathrm{~h}$ and (d, e, f) $48 \mathrm{~h}$ growth at different glucose concentrations (1, $5.5 \mathrm{mmol} / \mathrm{l} ; 2,11.1 \mathrm{mmol} / \mathrm{l}$ and 3 $16.5 \mathrm{mmol} / \mathrm{l})$. The trials were cells alone (control), treated with insulin $(100 \mathrm{nmol} / \mathrm{l})$ or metformin $(10 \mathrm{mmol} / \mathrm{l})$ in the absence $($ white bars) or presence (black bars) of $10 \mu \mathrm{mol} / \mathrm{l} \gamma$-conglutin. The tests were performed in Dulbecco's minimum essential medium supplemented with $0.2 \%$ bovine serum albumin as detailed under 'Materials and methods' section. Values are means, with their standard errors represented by vertical bars of three independent experiments, each performed in quadruplicate. ${ }^{*}$ Mean values were significantly different from those of the $\gamma$-conglutin untreated trials $(P<0.05)$. 
of its proteins, can provide an alternative to the drug treatment of glucose metabolism disorders. More specifically, lupin flours, such as other pulses, are characterised by a lowglycaemic index, so they can be useful in the prevention of IR in human subjects ${ }^{(25)}$. Lupin seeds are characterised by a high content of protein, about $35 \%$, and by low levels of isoflavones and anti-nutritional factors ${ }^{(26)}$. The anti-diabetic activity of toasted lupin seeds was first described, in the middle of the last century, by Ferranini \& Pirolli ${ }^{(27)}$ and by Orestano $^{(28)}$, who proposed lupin as a substitute for the insulin therapy in mild-to-medium diabetes mellitus, but no further studies have been carried out to identify the molecule responsible of this biological effect. A few years ago, the potential of a single oral administration of $\gamma$-conglutin, a lupin seed protein, was demonstrated by Magni et al. ${ }^{(13)}$ in rats after glucose overload. In that study, the interaction of the lupin protein with insulin was also shown. However, the role of this interaction has not been elucidated so far.

In the present study, the $10 \%$ glucose supplied in drinkingwater induced hyperglycaemia and hyperinsulinaemia in rats similar to that observed in human subjects. No side effects have been detected during the experimental period, such as those recorded in animals undergoing streptozotocin ${ }^{(29)}$ or alloxan $^{(30)}$ treatment to mimic diabetes. $\gamma$-Conglutin administration has been demonstrated to counteract the plasma glucose increase as well as to improve the insulin sensitivity, normally reduced by the glucose-rich drinking-water. In the $\gamma$-conglutin-treated rats, the insulin sensitisation was increased significantly, as indicated by the $48 \%$ reduction in the homeostasis model of IR. It is worth noting that the hypoglycaemic effect in vivo was obtained by the use of a preparation, which contained a $\gamma$-conglutin amount corresponding to the lowest dose previously used in acute trials of glucose overload $^{(13)}$. Moreover, lower glucose levels were detected in $\gamma$-conglutin-treated rats following oral glucose overload; these results were confirmed by lower glycaemia in fasting and $2 \mathrm{~h}$ postprandial conditions. The mechanism/s, underlying the present results, are currently under investigation, but the hypothesis that $\boldsymbol{\gamma}$-conglutin could act as an insulin-like agent should not be excluded. Recently, Terruzzi et al. ${ }^{(31)}$ have shown that $\gamma$-conglutin may regulate muscle energy metabolism, protein synthesis and major histocompatibility complex gene transcription through the modulation of the same insulin signaling pathway. Moreover, $\boldsymbol{\gamma}$-conglutin resistance to proteases at neutral $\mathrm{pH}$ values ${ }^{(32)}$ could explain the maintenance of its activity after $48 \mathrm{~h}$ of incubation, as we have observed in HepG2 cells. The role of $\boldsymbol{\gamma}$-conglutin in controlling glucose concentrations has been assessed with the purified protein in cell assays (the present study) and animal models in previous studies ${ }^{(13)}$; however, the synergic effect of other protein/peptide components in the type F sample cannot be excluded. In addition, the reduced increase in plasma glucose $(-24 \%)$ and insulin $(-33 \%)$ levels, recorded in rats following $\gamma$-conglutin treatment $v$. the values found in the pair-fed animals, might be of pharmacological relevance. It is noteworthy, in fact, that these decreases have been obtained by the use of a single daily administration of a purified food protein. Although, these data need to be confirmed by human studies, the potential of lupin seed $\gamma$-conglutin to control glycaemia could be considered before developing new therapeutic strategies for the prevention or regression of glucose metabolism modifications.

The linkages between diet and health are no longer a matter of discussion. Moreover, the specific and/or limited effects of current drug treatments for diabetes, combined with dangerous side effects that most of them induce, have fueled the search for alternative medicine. At this point, the anti-diabetic effects of cinnamaldehyde and berberine in rats with type 2 diabetes, involving the up-regulation of tissue retinol-binding protein 4-GLUT4 protein, have been published by Zhang et al. ${ }^{\text {(33) }}$.

Furthermore, the specific role of many food components, their synergies and antagonism are still a largely unexplored area. The case of dietary proteins/peptides is particularly intriguing due to the dramatic changes they may undergo from food production to food digestion. On the other hand, the hypothesis that peptides with biological/pharmacological activities can be produced in vivo have been the object of recent papers ${ }^{(34)}$. Other food proteins, such as cod and soya proteins, improved glucose tolerance and insulin sensitivity in rats ${ }^{(35)}$, compared with casein; this effect seems due to the different amino acid composition of these proteins, which could be responsible for insulin's diminished ability to stimulate peripheral glucose transport. At present, we do not know whether $\gamma$-conglutin would be able, in vivo, to interfere in the production/secretion of glucagon-like peptide 1 or/and glucose-dependent insulinotropic peptide, thus acting as incretino-mimetic compound ${ }^{(36)}$. Further studies, aimed at understanding the protein moiety of $\gamma$-conglutin responsible for the glucose-lowering effect and the molecular mechanism thereby, are currently being undertaken.

In conclusion, the present study provides the in vivo and in vitro evidence of the involvement of $\gamma$-conglutin on cell glucose homeostasis, thus suggesting the potential use of this food protein in the control of glycaemia in patients with manifest or pre-clinical diabetes as well as for applications as functional foods and dietary supplements.

\section{Acknowledgements}

The present study was partially supported by a grant of MIUR (cod. RBAU01JS5C_004) from Italy. M. R. L. and Cr. M. designed the study; S. C., A. P., Cr. M. and C. M. conducted the study; M. R. L., M. D., Cr. M. and C. M. analysed the data; M. R. L. and M. D. wrote the manuscript and had primary responsibility for final content. All authors read and approved the final manuscript. The authors declare that there are no conflicts of interest.

\section{References}

1. Lovati MR, Manzoni C, Gianazza E, et al. (2000) Soy protein peptides regulate cholesterol homeostasis in HepG2 cells. J Nutr 130, 2543-2549.

2. Duranti M, Lovati MR, Dani V, et al. (2004) The $\alpha^{\prime}$ subunit from soybean $7 \mathrm{~S}$ globulin lowers plasma lipids and 
up-regulates VLDL-liver receptors in rats fed a hypercholesterolemic diet. J Nutr 134, 1334-1339.

3. Castiglioni S, Manzoni C, D'Uva A, et al. (2003) Soy proteins reduce progression of a focal lesion and lipoprotein oxidability in rabbits fed on a cholesterol-rich diet. Atherosclerosis 171, 163-170.

4. Sirtori CR, Lovati MR, Manzoni C, et al. (2004) Proteins of white lupin seed, a naturally isoflavone-poor legume, reduce cholesterolemia in rats and increase LDL receptor activity in HepG2 cells. J Nutr 134, 18-23.

5. Manzoni C, Duranti M, Eberini I, et al. (2003) Subcellular localization of soybean $7 \mathrm{~S}$ globulin in HepG2 cells and LDL receptor up-regulation by its $\alpha^{\prime}$ constituent subunit. J Nutr 133, 2149-2155.

6. Consonni A, Lovati MR, Manzoni C, et al. (2009) Cloning, yeast expression, purification and biological activity of a truncated form of the soybean $7 \mathrm{~S}$ globulin $\alpha^{\prime}$ subunit involved in HepG2 cell cholesterol homeostasis. J Nutr Biochem; 21, 887-891.

7. Cho SJ, Juillerat MA \& Lee CH (2008) Identification of LDLreceptor transcription stimulating peptides from soybean hydrolysate in human hepatocytes. J Agric Food Chem 56, $4372-4376$

8. Scarafoni A, Magni C \& Duranti M (2007) Molecular nutraceutics as a mean to investigate the positive effects of legume seed proteins on human health. Trends Food Sci Technol 18, 454-463.

9. Restani P, Duranti M, Cerletti P, et al. (1981) Subunit composition of the seed globulins of Lupinus albus. Phytochem 20, 2077-2083.

10. Blagrove RJ, Gillespie JM, Lilley GG, et al. (1980) Physicochemical studies on conglutin $\gamma$, a storage globulin from seeds of Lupinus angustifolius. Aust J Plant Physiol 7, 1-13.

11. Kagawa H, Yamauchi F \& Hirano H (1987) Soybean basic $7 S$ globulin represents a protein widely distributed in legume species. FEBS Lett 226, 145-149.

12. Satoh S, Sturm A, Fuji T, et al. (1992) cDNA cloning of an extracellular dermal glycoprotein of carrot and its expression in response to wounding. Planta 188, 432-438.

13. Magni C, Sessa F, Accardo E, et al. (2004) Conglutin $\gamma$, a lupin seed protein, binds insulin in vitro and reduces plasma glucose levels of hyperglycemic rats. J Nutr Biochem 15, 646-650.

14. Hanada K \& Hirano H (2004) Interaction of a 43-kDa receptor-like protein with a $4-\mathrm{kDa}$ hormone-like peptide in soybean. Biochemistry 43, 12105-12112.

15. Midaoui AE, Elimadi A, Wu L, et al. (2003) Lipoic acid prevents hypertension, hyperglycemia, and the increase in heart mitochondrial superoxide production. Am J Hypertens 16, 173-179.

16. Bez J, Schott M, Knauf U, et al. (2005) Protein isolates from sweet white lupin seeds: pilot production and techno-functional properties. In Optimized Processing for Preparing Healthy and Added Value Food Ingredients from Lupin Kernel, the European Protein-rich Grain Legume, pp. 21-35 [A Arnoldi, editor]. Rome: Aracne Editrice SRL.

17. Duranti M, Scarafoni A, Gius C, et al. (1994) Heat induced synthesis and tunicamycin-sensitive secretion of the putative storage glycoprotein conglutin $\gamma$ from mature lupin seeds. Eur J Biochem 222, 387-393.
18. Capraro J, Spotti P, Magni C, et al. (2010) Spectroscopic studies on the pH-dependent structural dynamics of $\gamma$-conglutin, the blood glucose-lowering protein of lupin seeds. Int J Biol Macromol 47, 502-507.

19. Laemmli UK (1970) Cleavage of structural proteins during the assembly of the head of bacteriophage T4. Nature 227, 660-665.

20. Midaoui AE \& De Champlain J (2002) Prevention of hypertension, insulin resistance, and oxidative stress by $\alpha$-lipoic acid. Hypertension 39, 303-307.

21. Li YY, Wu HS, Tang L, et al. (2007) The potential insulin sensitizing and glucose lowering effects of a novel indole derivative in vitro and in vivo. Pharmacol Res 56, 335-343.

22. Duranti M, Consonni A, Magni C, et al. (2008) The major proteins of lupin seed: characterisation and molecular properties for use as functional and nutraceutical ingredients. Trends Food Sci Technol 19, 624-633.

23. King H, Aubert RE \& Herman WH (1998) Global burden of diabetes, 1995-2025: prevalence, numerical estimates, and projections. Diabetes Care 21, 1414-1431.

24. Parikh N, Pencina M, Wang T, et al. (2007) Increasing trends in incidence of overweight and obesity over 5 decades. Am J Med 120, 242-250.

25. Duranti M (2006) Grain legume proteins and nutraceutical properties. Fitoterapia 77, 67-82.

26. Champ M (2001) Benefits of pulses in human diet. In Proceedings of 4th European Conference on Grain Legumes, pp. 109-113. Valladolid: AEP.

27. Ferranini A \& Pirolli M (1937) L'azione del decotto di semi di Lupinus albus sulla curva glicemica da carico di glucosio nei soggetti normali e diabetici. Folia Med 23, 729-748.

28. Orestano G (1940) Sull'azione ipoglicemica dei semi di Lupinus albus. Arch Farmacol Sperim 70, 113-117.

29. Kordowiak AM, Dudek B \& Grybo R (2004) Influence of sodium bis (oxalato)oxovanadate (IV) on phospholipids in liver Golgi fraction from control and streptozotocin-diabetic rats. Comp Biochem Physiol C Toxicol Pharmacol 125, $11-16$.

30. Pari L \& Saravanan G (2002) Antidiabetic effect of Cogent db, a herbal drug in alloxan-induced diabetes mellitus. Comp Biochem Physiol C Toxicol Pharmacol 131, 19-25.

31. Terruzzi I, Senesi P, Magni C, et al. (2009) Insulin-mimetic action of conglutin- $\gamma$, a lupin seed protein, in mouse myoblasts. Nutr Metab Cardiovasc Dis 21, 197-205.

32. Capraro J, Magni C, Scarafoni A, et al. (2009) Susceptibility of lupin $\gamma$-conglutin, the plasma glucose-lowering protein of lupin seeds, to proteolytic enzymes. J Agric Food Chem 57, 8612-8616.

33. Zhang W, Xu YC, Guo FJ, et al. (2008) Anti-diabetic effects of cinnamaldehyde and berberine and their impacts on retinolbinding protein 4 expression in rats with type 2 diabetes mellitus. Chin Med J 121, 2124-2128.

34. Lovati MR, Manzoni C, Castiglioni S, et al. (2006) Soy peptide and cholesterol homeostasis. FASEB J 18, A86.

35. Lavigne C, Marette A \& Jacques H (2000) Cod and soy proteins compared with casein improve glucose tolerance and insulin sensitivity in rats. Am J Physio Endocrinol Metab 278, E491-E500.

36. Nicolucci A \& Rossi MC (2008) Incretin-based therapies: a new potential treatment approach to overcome clinical inertia in type 2 diabetes. Acta Biomed 79, 184-191. 